\title{
Effect Analysis of Different Combustion Models on the Combustion in Foursquare Tangential Circle Boiler by Numerical Simulation
}

\author{
Lei Du a, Yue Guo ${ }^{\text {b }}$, Long Jiang ${ }^{c}$, Qing Li ${ }^{d}$, Zhenning Zhao ${ }^{e}$ \\ North China Electric Power Research Institute Co Ltd. Beijing, 100045, China. \\ adulei426311@163.com, bhosa@163.com, ckaveykikiy@163.com, d13910680082@139.com, \\ ezhaozn@sina.com
}

\begin{abstract}
The mixture fraction/probability density function (PDF) model and eddy break up (EBU) model were used to simulate the combustion in a 200MW foursquare tangential circle boiler, calculating the flue gas velocity field, temperature field and combustion product concentration distribution in the furnace. The calculated outlet oxygen concentration and coal burning rate via EBU model were $2.7 \%$ and $98.38 \%$, and were $3.0 \%$ and $98.82 \%$ for PDF model; the convergence time of EBU model is half of PDF model.
\end{abstract}

Keywords: Pulverized coal boiler; mixture fraction/probability density function model; eddy break up model; numerical simulation.

\section{Introduction}

Computational fluid dynamics (CFD), as a new research approach, was widely applied to predict the reaction flow condition and provided an effective way to research the coal-fired boiler with less cost. In this paper, the combustion process in a 200MW foursquare tangential circle boiler was simulated via CFD for researching the turbulent flow, particle transport and combustion, gas phase reaction and radiation heat transfer in the furnace [1]. The specific calculation models adopted in CFD were mixture fraction/probability density function (PDF) model and eddy break up (EBU) model, obtaining the required convergence time, the flue gas velocity field, temperature field and the concentration distribution of combustion products in the furnace.

\section{Overview of boiler}

The parameters of the selected boiler contain: the type of WGZ670/13.7-8, foursquare tangential combustion, subcritical parameter, single furnace, single reheating, balanced ventilation, solid state slag drum boiler, the width, depth and height of the furnace were $11.92 \mathrm{~m}, 10.80$ and 45.40 respectively, intermediate storage type hot air feeding system. Each corner of the burner area is arranged with 4 primary air nozzles, 6 secondary air nozzles, 2 third air nozzles, 2 blast furnace gas (BFG) and 2 coke oven gas (COG) nozzles. The BFG nozzle is arranged at the bottom part, and the COG nozzle is arranged in the secondary air nozzle. The primary air, secondary air and BFG adopt double tangential circle system, but the third air adopts collisional system with the angle of 10 degrees. The furnace size and combustion system structure are shown in Fig. 1. The burning coal is a kind of meagre coal and the maximum BFG and COG flow are 250000 and $24000 \mathrm{Nm}^{3} / \mathrm{h}$ respectively. 


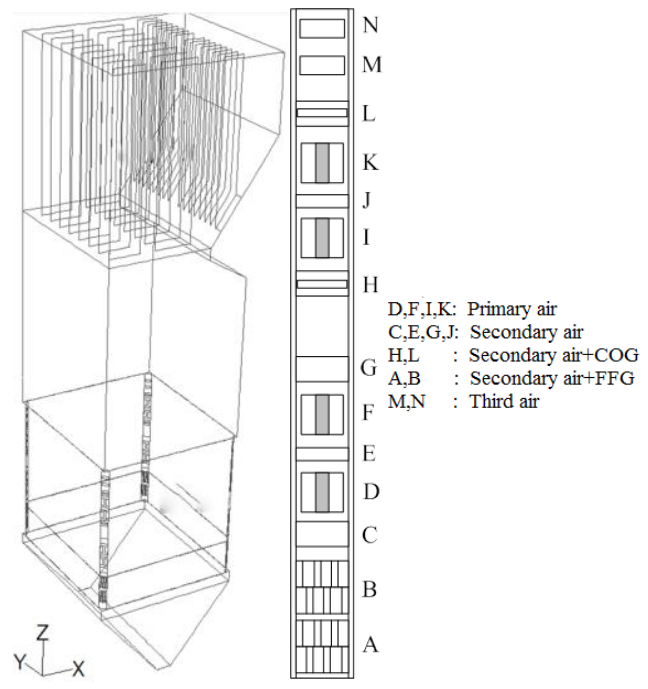

Fig. 1 Schematic diagram of furnace size and combustion system structure

The elemental analysis and industrial analysis of the burning coal for boiler are shown in Table 1 . The low calorific value of burning coal is $19100 \mathrm{~kJ} / \mathrm{kg}$.

Table 1 elements and industrial analysis of burning coal

\begin{tabular}{ccccccccc}
\hline & \multicolumn{3}{c}{ Elemental analysis $(a d, \%)$} & \multicolumn{4}{c}{ Industry analysis $(a d, \%)$} \\
\hline$C$ & $H$ & $O^{*}$ & $N$ & $S$ & $M$ & $V$ & $A$ & $F c$ \\
54.62 & 2.80 & 5.52 & 0.88 & 0.49 & 1.58 & 12.80 & 35.74 & 49.88 \\
\hline
\end{tabular}

The coal particles diameters were selected according to the Rosin-Rammler method: the minimum particle diameter is $5 \mu \mathrm{m}$; the maximum particle diameter is $250 \mu \mathrm{m}$; the average particle diameter is $65 \mu \mathrm{m}$; the distribution index is 1.5 .

\section{Mathematical models and computational conditions}

\subsection{Mathematical model}

We will use the Fluent 6.3.26 to carry out the research, the gas flow in a boiler furnace for three dimensional turbulent flow reaction, its average flow to be considered as steady flow, by using the standard k- $\varepsilon$ turbulence model. The mixed scores/probability density function model is used to simulate the gas phase turbulent combustion, the pulverized coal particle stochastic trajectory model is used to simulate motion; the double-equation parallel reaction model is used in the coal pyrolysis, coke combustion is controlled by power/diffusion reaction rate model, the method of P1 is used to the radiation heat transfer calculations.

The air flow in pulverized coal furnace is a three-dimensional turbulent reacting flow, which can be regarded as a steady state [2]. In the three-dimensional Cartesian coordinate system, the conservative equation of turbulent reacting flow is listed as follow [3]:

$$
\frac{\partial}{\partial x}(\rho u \phi)+\frac{\partial}{\partial y}(\rho v \phi)+\frac{\partial}{\partial z}(\rho w \phi)=\frac{\partial}{\partial x}\left(\Gamma_{\phi} \frac{\partial \phi}{\partial x}\right)+\frac{\partial}{\partial y}\left(\Gamma_{\phi} \frac{\partial \phi}{\partial y}\right)+\frac{\partial}{\partial z}\left(\Gamma_{\phi} \frac{\partial \phi}{\partial z}\right)+S_{\phi}+S_{p \phi}
$$

where: $\rho$ is fluid density; $\phi$ is universal variables; $\mathrm{m}$ is quality; $\mathrm{u}, \mathrm{v}$ and $\mathrm{w}$ are the three component of velocity; $\mathrm{p}$ is pressure; $\kappa$ is turbulent kinetic energy; $\varepsilon$ is dissipation rate; $\mathrm{f}$ is component fraction; $\mathrm{g}$ is pulse mean square value; $h$ is enthalpy; gas source; $S \phi$ is the source of air; $S p \phi$ is the source of solid particles; $\Gamma \phi$ is diffusion coefficient.

The mixture fraction/probability density function (PDF) model and eddy break up (EBU) model were used to simulate the gas phase turbulent combustion [4]. The eddy dissipation model proposes that the fuel and oxidant are distributed in different vortex, and the burning rate is decided by the mixing rate at the molecular scale between fuel vortex and oxidizer vortex, namely the eddy dissipation rate. Because the chemical reaction process is faster than the turbulent transport process, the combustion process is mainly controlled by the chemical reaction process [5]. The reaction paths 
of the pulverized coal volatilization are listed as follow, and the values of $\alpha, \beta, \gamma$ are 2.056, 1.136, 2.232 [6].

The reaction paths of the matter (2) and (3) show that the alpha, beta, gamma are 2.056, 1.136 and 2.232 respectively.

Volatile matter $+\alpha \mathrm{O}_{2} \rightarrow \beta \mathrm{CO}+\gamma \mathrm{H}_{2} \mathrm{O}$

$\mathrm{CO}+0.5 \mathrm{O}_{2} \rightarrow \mathrm{CO}_{2}$

The standard k- $\varepsilon$ turbulence model was adopted in this paper: the pulverized coal particles with stochastic trajectory model was used for simulating the particle movement; the single equation model for coal pyrolysis; kinetics/diffusion controlled reaction rate model for coke combustion; P1 method for radiative heat transfer. The detailed descriptions of these models are shown in the literature [5].

\subsection{Computational conditions}

The solution area model was built based on the structure size of boiler furnace, and the area is divided via the regional meshing method. For the burner outlet region, the paving style was used to increase the density for simulating the flow characteristics of the outline accurately, as shown in Fig. 2 .

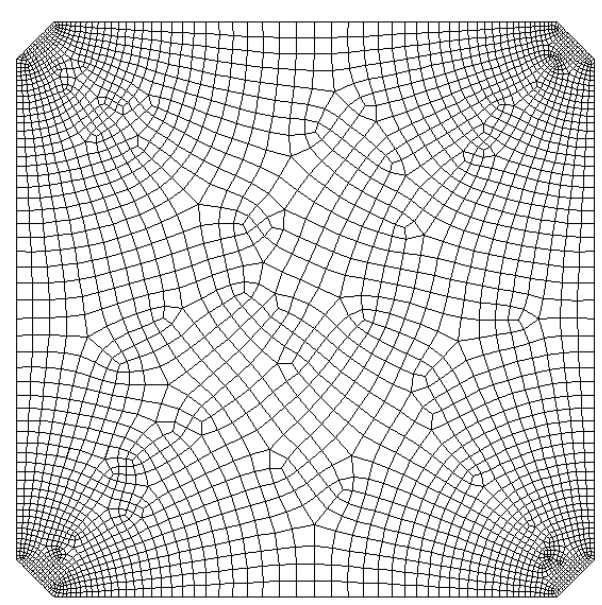

Fig. $2 \mathrm{Z}$ section of burner area

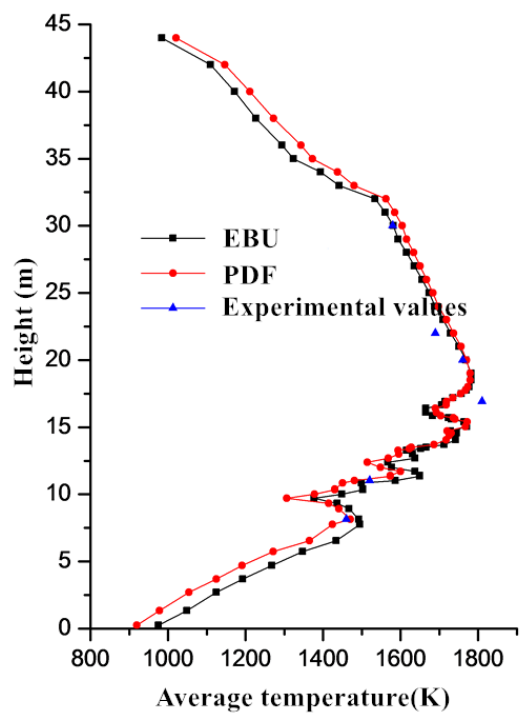

Fig. 3 Comparison between the calculated and measured average temperature along the furnace height

The entrance velocity and temperature were set according to the operation parameters and the entrances of burner and overfire air. The outlet used the pressure outlet boundary condition (-50 Pa). The surface temperature employs no slip temperature boundary condition and the given surface temperature and the radiation rate are $698 \mathrm{~K}$ and 0.6. The pre-exponential factor of the kinetic parameters of volatile precipitation is $49200 \mathrm{~s}^{-4}$ and the activation energy is $74000 \mathrm{~kJ} / \mathrm{kmol}$; the 
pre-exponential factor of the kinetic parameters of char combustion is $0.0016 \mathrm{~kg} / \mathrm{m}^{2} . \mathrm{s} . \mathrm{Pa}$ and the relative activation energy is $83700 \mathrm{~kJ} / \mathrm{kmol}$.

The whole simulation was carried out under the full load of 200MW: for working condition 1, the EBU model was used; for working condition 2, the PDF model was used; the specific calculated inlet boundary conditions are shown in Table 2 .

Table 2 Boundary conditions for the calculation

\begin{tabular}{cccc}
\hline Nozzles & air speed $(\mathrm{m} / \mathrm{s})$ & $\begin{array}{c}\text { air } \\
\text { temperature }\left({ }^{\circ} \mathrm{C}\right)\end{array}$ & $\begin{array}{c}\text { hydraulic } \\
\text { diameter }(\mathrm{m})\end{array}$ \\
\hline primary air & 24 & 282 & 0.4695 \\
secondary air & 55 & 362 & 0.4667 \\
Third air & 23 & 80 & 0.2635 \\
\hline
\end{tabular}

The air speed in each nozzle was set based on the actual measured value and air distribution uniformity for all nozzles. The amount of powdered coal carried by the third air is $1.072 \mathrm{~kg} / \mathrm{s}$. Three coal milling machine (A, C, D) operated during the experiment.

When calculating the iteration, the flow field with a certain degree of convergence was obtained by 600 times of the cold state calculation ( $1.5 \mathrm{~h}$ for two models), then, the thermal state was calculated until they converge ( $25 \mathrm{~h}$ for EBU and $50 \mathrm{~h}$ for PDF model). Two working conditions both adopted the single core operation. The SIMPLE algorithm was used to solve the pressure and velocity coupling of discrete equations; the linear iteration method and low relaxation factor was applied to solve equations. The convergence criteria contain: energy, radiation heat transfer, the calculation of the residual error is less than $1^{*} 10^{-6}$, and the residual error of other items is less than $1^{*} 10^{-3}$.

\section{Results and Analysis}

\subsection{Simulation results validation}

In order to verify the rationality of the simulation results, the boiler combustion test was carried out under the same load condition. The average furnace temperature along the furnace height was measured via the flame temperature measurement system [7].

From Fig. 3 (comparison of the calculated and measured average temperature along the height of boiler furnace) we can see: the calculated temperatures are in agreement with the experimental values; the maximum calculated error of average temperature for two working conditions are $4.52 \%$ and $5.46 \%$. The conclusions obtained above present that: the selected mathematical model and grid can reasonably predict the flow, combustion and heat transfer characteristics in the boiler; the EBU and FDB models can be used to simulate the combination process of the pulverized coal in furnace.

\subsection{Average oxygen concentration}

Fig. 4 presents the average oxygen concentration along the furnace height. Compared with Fig. 3, the distribution among temperature field, velocity field and oxygen concentration distribution have a good agreement. In the main combustion region, the average oxygen concentration increases sharply firstly because the primary and secondary winds carry a large amount of oxygen into the furnace, then the oxygen concentration declines sharply due to the rapid consumption of oxygen after the ignition of pulverized coal. Because more oxygen is consumed in the main combustion zone of EBU model, the descending trend of average oxygen concentration in upper boiler furnace is slower than PDF model with the burnout of pulverized coal. The calculated outlet oxygen concentration and coal burning rate by EBU model are $2.7 \%$ and $98.38 \%$, and for PDF are $3.0 \%$ and $98.82 \%$ 


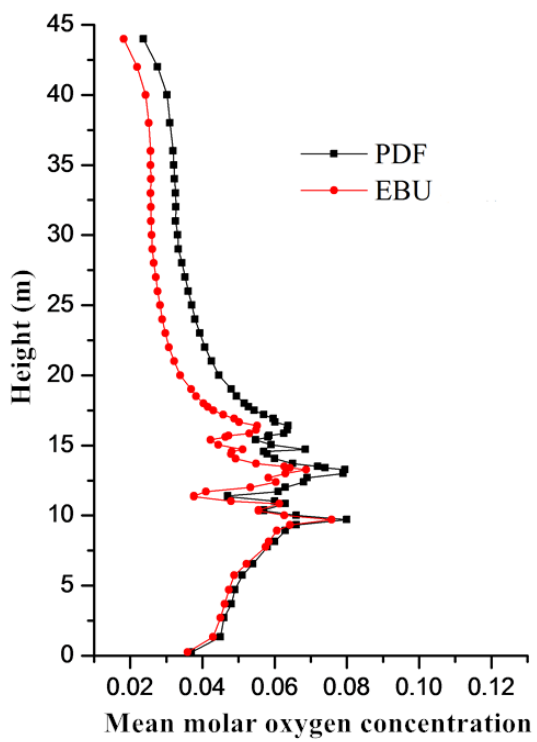

Fig. 4 Comparison of calculated average oxygen concentration along the furnace height

\section{Conclusion}

(1) By comparing the calculated and measured average temperature along the furnace height, the adopted mathematical model and the grid can reasonably predict the flow, combustion and heat transfer characteristics of the boiler; meanwhile, the mixture fraction/probability density function model and eddy break up model can be used to simulate the combination of the pulverized coal in furnace.

(2) The calculated outlet oxygen concentration and coal burning rate by EBU model were $2.7 \%$ and 98.38\%, and for PDF were $3.0 \%$ and $98.82 \%$.

The calculated oxygen concentration in output

the export EBU model to calculate the oxygen concentration is $2.7 \%$, the coal burnout rate is $98.38 \%$, the export PDF model to calculate the oxygen concentration is $3 \%$, the coal burnout rate is $98.82 \%$;

(3) The time required for convergence of the EBU model is half of PDF model.

\section{References}

[1] Soot, L. D. and P. J. Smith, Coal combustion and gasification. 1985: Springer.

[2] Zhou L. X. Theoretical and numerical simulation of the flow and combustion of turbulent gas and particle. 1994: Science Press.

[3] Pa T.K., Zhang Z. Numerical calculation of heat transfer and fluid flow. 1984: Science Press.

[4] Su S. Simulation of coal blended combustion with the two-mixture-fraction/PDF method. Proceedings of the Chinese Society for Electrical Engineering, 2012. 2.

[5] Sheng, C, A computational fluid dynamics based study of the combustion characteristics of coal blends in pulverised coal-fired furnace. Fuel, 2004. 83(11-12): p. 1543-1552.

[6] Fang, Q, Numerical Simulation of Multi-Fuel Combustion in a 200 MW Tangentially-Fired Utility Boiler. Energy \& Fuels, 2012.

[7] Huajian, W., Measurements on flame temperature and its 3D distribution in a $660 \mathrm{MWe}$ arch-fired coal combustion furnace by visible image processing and verification by using an infrared pyrometer. Measurement Science and Technology, 2009. 20: p. 114006. 